Research Article

\title{
Canonical Wnt Signaling Pathway on Polarity Formation of Utricle Hair Cells
}

\author{
Di Deng $\mathbb{D}^{1,2}$ Xiaoqing Qian $\mathbb{D}^{2,3}$ Binjun Chen $\mathbb{D}^{2,3}$ Xiaoyu Yang $\mathbb{D}^{2,3}$ Yanmei Wang ${ }^{2,},{ }^{2,3}$ \\ Fanglu Chi $\mathbb{D}^{2,3}$ Yibo Huang $\mathbb{D}^{2,3}$ Yu Zhao $\mathbb{D}^{1},{ }^{1}$ and Dongdong Ren $\mathbb{D}^{2,3}$ \\ ${ }^{1}$ Department of Otolaryngology, Head and Neck Surgery, West China Hospital, Sichuan University, Chengdu 610041, China \\ ${ }^{2}$ NHC Key Laboratory of Hearing Medicine (Fudan University), Shanghai 200031, China \\ ${ }^{3}$ ENT Institute and Department of Otorhinolaryngology, Eye \& ENT Hospital, Fudan University, Shanghai 200031, China
}

Correspondence should be addressed to Yibo Huang; adabobohuang@126.com, Yu Zhao; yutzhao@163.com, and Dongdong Ren; dongdongren@fudan.edu.cn

Received 31 March 2021; Revised 26 April 2021; Accepted 11 May 2021; Published 24 May 2021

Academic Editor: Renjie Chai

Copyright (C 2021 Di Deng et al. This is an open access article distributed under the Creative Commons Attribution License, which permits unrestricted use, distribution, and reproduction in any medium, provided the original work is properly cited.

As part of the inner ear, the vestibular system is responsible for sense of balance, which consists of three semicircular canals, the utricle, and the saccule. Increasing evidence has indicated that the noncanonical Wnt/PCP signaling pathway plays a significant role in the development of the polarity of the inner ear. However, the role of canonical Wnt signaling in the polarity of the vestibule is still not completely clear. In this study, we found that canonical Wnt pathway-related genes are expressed in the early stage of development of the utricle and change dynamically. We conditionally knocked out $\beta$-catenin, a canonical Wnt signaling core protein, and found that the cilia orientation of hair cells was disordered with reduced number of hair cells in the utricle. Moreover, regulating the canonical Wnt pathway (Licl and IWP2) in vitro also affected hair cell polarity and indicated that Axin2 may be important in this process. In conclusion, our results not only confirm that the regulation of canonical Wnt signaling affects the number of hair cells in the utricle but also provide evidence for its role in polarity development.

\section{Introduction}

The cochlea and vestibule are important for hearing and balance, respectively. They detect sound and position signals through stereociliary bundle on the surface of hair cells (HCs) [1-4]. The vestibular system contains utricular maculae, saccular maculae, and three semicircular canal cristae. Planar polarity of the stereociliary bundle guarantees that the range of motion is detected properly by vestibular HCs [5]. In the inner ear, the stereocilia are arranged in an ascending cluster, with the highest being located adjacent to the kinocilium $[6,7]$. The direction of stereociliary bundle between adjacent HCs are highly similar. Compared to the cochlea, an artificial line (line of polarity reversal, LPR) passes through the maculae. The maculae are divided into two regions: the kinocilium of the utricular maculae faces the LPR, while the kinocilium of the saccular maculae points away from the LPR [8].
In the canonical Wnt pathway, Wnt protein binds to Frizzled on the cell surface to inhibit the activity of the $\beta$ catenin-degrading complex composed of GSK-3, APC, and Axin. $\beta$-Catenin accumulates in the cytoplasm and is then transferred to the nucleus, where it controls embryogenesis and cell fate through T-cell factor/lymphoid enhancerbinding factor (TCF/LEF). The canonical Wnt signaling pathway and its downstream target genes play an important regulatory role in cell proliferation [9-13] and differentiation [14-18] during inner ear development [19], as well as in the survival of HCs [20-24] and spiral ganglion neurons [25]. During development, the expression of Wnt signaling significantly decreased with age in the inner ear [26]. The canonical Wnt pathway is essential to induce Pax2-positive otic placode cells to form an auditory sac [27]. In our previous studies, we found that polarity of striola in the medial region occurred earlier than in the lateral region, consistent with proliferation and differentiation in utricle HCs [28]. This 
led us to hypothesize that the canonical Wnt signaling pathway is involved in polarity development in the utricle. Here, we found differences in the expression of Wnt pathwayrelated genes among different stages of polar formation in the utricle. We conditionally knocked out $\beta$-catenin, a core protein in the canonical Wnt signaling pathway, in Sox2positive cells in mice. The number and polarity of HCs were also affected. Moreover, the polarity of the utricle was affected by treatment with an activator or inhibitor of the canonical Wnt pathway in vitro, and we found that Axin2 may be a key role in this process (Figure 1).

\section{Materials and Methods}

2.1. Animal Protocols. All protocols were approved by the Animal Care and Use Committee of the EENT Hospital of Fudan University. Healthy wild-type C57BL/6 mice were purchased from Shanghai SLAC Laboratory Animal Co., Ltd. (Shanghai, China). Female and male mice were mated in the same nest at $10 \mathrm{AM}$ and recorded as E0. They were separated at $10 \mathrm{AM}$ the next day and recorded as E0.5. In turn, E11.5 were recorded on day 12, E12.5 on day 13, and E13.5 on day 14. We also performed tap detection at E0.5.

Sox $2^{\text {CreER }}$ and Ctnnb1 ${ }^{\text {flox(exon2-6) }}$ mice were provided by Huawei Li at Fudan University and maintained in a mixed background of C57BL/6J and BALB/C. Standard PCR was performed to genotype transgenic mouse offspring. DNA was isolated by incubating the tail tip in a $100 \mu \mathrm{L}$ mixture of Direct PCR and Protein K $(100: 2)$ at $55^{\circ} \mathrm{C}$ overnight and then at $95^{\circ} \mathrm{C}$ for $1 \mathrm{~h}$. The primers used were as follows: GA486 (TGCCACGACCAAGTGACAGCAATG) and GA487 (ACCAGAGACGGAAATCCATCGCTC) for the Cre allele, with an expected fragment size of 300-400 bp; R15 (AAGGTAGAGTGATGAAAGTTGTT); and R16 (CAC CAT GTC CTC TGT CTA TTC) for the $\beta$-catenin knockout allele, with expected fragment sizes of $\sim 300$ and $223 \mathrm{bp}$ for the mutant and wildtype, respectively. To activate Cre and avoid premature abortion due to tamoxifen, a tamoxifen/progesterone mixture dissolved in corn oil (Sigma Aldrich, St. Louis, MO, USA; $1 \mathrm{~mL}$ corn oil : $10 \mathrm{mg}$ tamoxifen : $20 \mathrm{mg}$ progesterone) was injected intraperitoneally once a day for 2 consecutive days. The dose on the first day (E11.5) was $0.1 \mu \mathrm{g} / \mathrm{g}$ tamoxifen, and the dose was halved on the following day (E12.5).

2.2. Utricle Harvest. The pregnant mice were anesthetized and killed. The embryo were removed from the uterus and quickly placed into a cold solution of $1 \times$ phosphatebuffered saline (PBS; $\mathrm{pH}=7.4$ ). The utricle was dissected with the microscope. P1 mice were anesthetized and killed; then, the utricle was harvested, and the otolith was removed with the microscope and harvest the utricle.

2.3. Tissue Culture In Vitro. The freshly dissected utricle was placed in Dulbecco's modified Eagle's medium (DMEM)/F12 medium (20\% foetal bovine serum [FBS], B27 (1: 100), streptomycin $[100 \mathrm{U} / \mathrm{mL}])$. The Petri dishes were incubated at $37^{\circ} \mathrm{C}$, with a concentration of $5 \% \mathrm{CO}_{2}$ and humidity of 95\%. Dimethyl sulfoxide (DMSO), lithium chloride ( $\mathrm{LiCl})$, or IWP2 was added 24 hours later. We treated cultures in the activator group with the canonical Wnt pathway activator $\mathrm{LiCl}(2.5 \mu \mathrm{M} / \mathrm{mL})$. The canonical Wnt inhibitor IWP2 $(1.0 \mu \mathrm{M} / \mathrm{mL})$ was added to the inhibitor group. DMSO $(1.0 \mu \mathrm{M} / \mathrm{mL})$ was added to the control group. The final culture concentration depended on the growth of the utricle. The culture medium was replaced in full every 24 hours, and the tissue was removed and fixed on the day 5 after treatment. For all experiments, three biological replicates were used.

2.4. PCR and RT-qPCR. The reaction protocol for PCR was as follows: predenaturation at $94^{\circ} \mathrm{C}$ for $5 \mathrm{~min}, 32$ cycles of denaturation at $94^{\circ} \mathrm{C}$ for $1 \mathrm{~min}$, annealing at $55^{\circ} \mathrm{C}$ for $1 \mathrm{~min}$, elongation at $72^{\circ} \mathrm{C}$ for $1.5 \mathrm{~min}$, and a final elongation at $72^{\circ} \mathrm{C}$ for $10 \mathrm{~min}$.

RNA was extracted from the utricle using the RNeasy Micro Kit (Qiagen, Hilden, Germany), and cDNA was synthesized using the GeneAmp ${ }^{\circledR}$ PCR System 9700 (Applied Biosystems, Foster City, CA, USA) by reverse transcription: $42^{\circ} \mathrm{C}$ for $15 \mathrm{~min}$ and $85^{\circ} \mathrm{C}$ for $5 \mathrm{~s}$. The qPCR reaction protocol was as follows: $94^{\circ} \mathrm{C}$ for $30 \mathrm{~s}$, followed by 45 cycles at $94^{\circ} \mathrm{C}$ for $5 \mathrm{~s}$ and $60^{\circ} \mathrm{C}$ for $30 \mathrm{~s}$. Each sample was run in triplicate for analysis purposes. At the end of the PCR cycles, melting curve analysis was performed to validate the generation of the expected PCR product. The primer sequences were designed in the laboratory and synthesized by TsingKe Biotech (Beijing, China) based on the mRNA sequences obtained from the NCBI database, as follows:

GADPH (F: GCAAGGACACTGAGCAAGA; R:GGAT GGAAATTGTGAGGGAG), Apc (F: GCCTGGATGAG CCATTTATAC; R: AGTTTCATTCCCATTGTCGT), Wif1 (F: TTGTACCTGTGGATCGACG; R:GGCTTTCCTGAAA TCATGTGT), Camk2d (F: AGATCAAGGCCGGAGCTTA; R:CAGAGGCTGTGATACGTT), Fzd6 (F: CTTCAGTGG CCTGTATCTT; R:CCATGTCATCTCCCAGGT), Axin2 (F:AGAAGAGGAGTGGACGTGTG; R:AGCTGTTTCCG TGGATCTCA), Vangl2 (F:TCTCTGGCCCTGACACATTT; R:ACTGAGGAAGAGGGGAGACT), Prickle2 (F:ATGCCA CCTTCTTCCTCCTC; R:AGTAGGTGACAAATGGCCGA).

2.5. RNA-Sequencing (RNA-Seq) and Protein-Protein Interaction (PPI) Network. Total RNA was extracted from the utricle (E13.5 and P1,wild-type C57BL/6 mice) and then submitted to Otogenetics Corporation (Atlanta, GA, USA) for RNA-Seq assays. We assessed RNA integrity and purity using an Agilent Bioanalyzer (Agilent Technologies, Santa Clara, CA, USA). cDNA was generated from total RNA using the Clontech SMARTer PCR cDNA Synthesis Kit (Clontech Laboratories, Inc., Mountain View, CA USA, catalogue \#634891). Bioruptor (Diagenode, Inc., Denville, NJ, USA) was used to fragment cDNA, an Agilent Bioanalyzer was used for profiling, and SPRIworks HT (catalogue \#B06938; Beckman Coulter, Pasadena, CA, USA) was used to prepare the Illumina library. The quality, quantity, and size distribution of the Illumina library were determined using TapeStation (Agilent Technologies).

The DAVID database (https://david.ncifcrf.gov/) is a commonly used database for gene analyses. KEGG pathway 


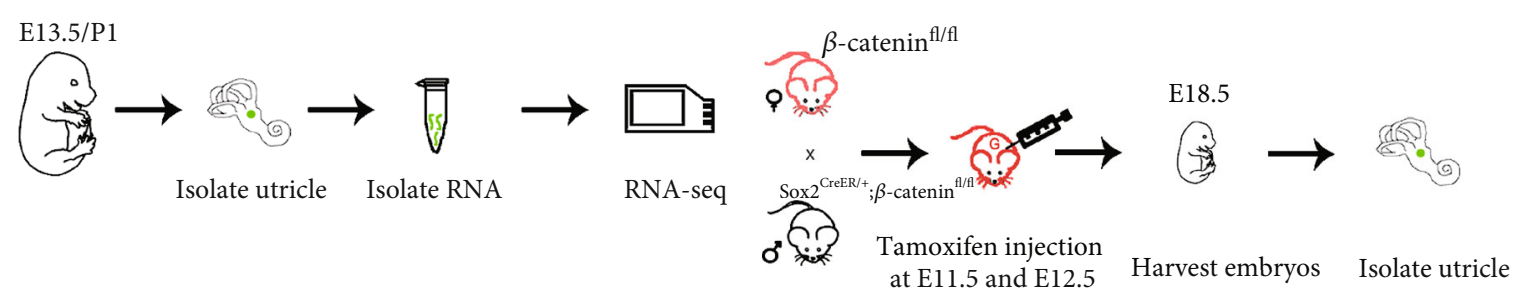

(a)

(b)

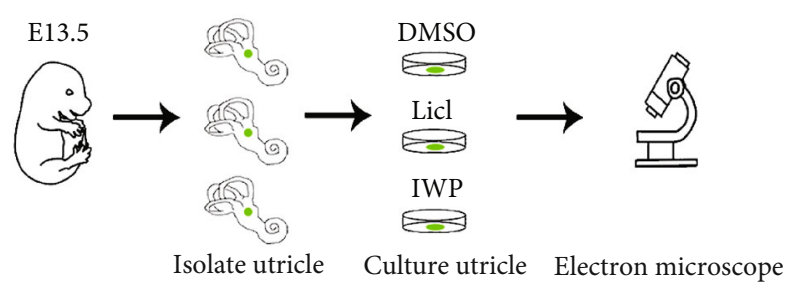

(c)

Figure 1: The experimental procedure. (a) RNA-sequencing of the utricle of E13.5 and P1 mice. (b) To conditionally knockout $\beta$-catenin, the $\beta$-catenin ${ }^{\mathrm{f} / \mathrm{f}}$ female mice were mated with $\beta$-catenin ${ }^{\mathrm{t} / \mathrm{f}}$ male mice with Sox $2^{\mathrm{CreER}+}$; the pregnant mice were treated with tamoxifen injection at E11.5 and E12.5, and the utricle of the embryonic mice was harvested at E18.5. (c) Utricles of E13.5 mice were cultured in vitro with DMSO (control), LiCl (activator), and IWP2 (inhibitor).

enrichment analyses were performed using DAVID, and the TXT files of the results were downloaded for subsequent analysis. For the Bonferroni test, $P$ values $<0.05$ were set considered significant, and $\log 2$ (fold_change) values between -1 and 1 were excluded from the analysis. STRING (https://string-db.org/) was used to analyse the interactions between proteins. Many signaling pathways play important roles in the development of the utricle. To better understand the role of the Wnt signaling pathway, we only included the Wnt pathway-related genes in this analysis.

2.6. Electron Microscope. Utricles were fixed with $2.5 \%$ glutaraldehyde at $4{ }^{\circ} \mathrm{C}$ overnight and then processed using $2 \%$ tannin and $1 \%$ osmium acid. Graded ethanol series were used to dehydrate the samples, and liquid $\mathrm{CO}_{2}$ (EM CPD300; Leica, Wetzlar, Germany) was used to dry them. An E-1045 sputter coater (Hitachi, Tokyo, Japan) was used to coat specimens with $100 \AA$ Au. A NOV A NanoSEM 230 scanning electron microscope (FEI, Hillsboro, OR, USA) was used to scan the samples.

2.7. Whole-Mount Immunostaining. Mouse embryos were harvested at E18.5. Otocysts were dissected in cold PBS and fixed with $4 \%$ paraformaldehyde (Electron Microscopy Services, Hatfield, PA, USA) for $1 \mathrm{~h}$ at $4^{\circ} \mathrm{C}$. After washing with PBS, utricles were dissected and blocked with $10 \%$ donkey serum, and $0.1 \%$ Triton-X in PBS for $30 \mathrm{~min}$ at room temperature, followed by incubating with primary antibodies diluted in PBS containing 5\% donkey serum and $0.1 \%$ Triton-X overnight at $4^{\circ} \mathrm{C}$. The next day, after washing extensively with PBS, tissues were incubated with secondary antibodies at $1: 1,000$ dilution in $0.1 \%$ Triton$\mathrm{X} 100$ (in PBS) for $2 \mathrm{~h}$ at room temperature. After extensive PBS washing, samples were mounted in antifade Fluorescence Mounting Medium (Agilent Technologies) on coverslips. We used mouse anti- $\beta$-spectrin $(1: 500$; catalogue \#612562; BD Biosciences) primary antibody and Alexa Fluor 568 Donkey Anti-Mouse IgG (1:1,000; Invitrogen, Carlsbad, CA, USA) secondary antibody. FITCconjugated phalloidin $(1: 1,000$; Invitrogen $)$ was used to visualize actin in the stereocilia of sensory HCs.

2.8. Quantification and Statistical Analyses. Whole-mount immunostaining images were acquired using a Zeiss LSM800 confocal microscope (Zeiss, Oberkochen, Germany) at $40 \times$ magnification, and the whole utricle image was obtained by flattening the overlapping parts of original images using Photoshop CS4 (Adobe Systems). The overall outline of a utricle was drawn on the stitched confocal image, and the area of the sensory epithelia was measured using the Zen image processing system (Zeiss). The number of HCs on the composite image was counted manually using Photoshop CS4 (Adobe Systems). Cell number was scored in two regions divided by LPR, defined as lateral and medial region. Hair bundle orientation was scored in three $40 \times 80 \mu \mathrm{m}^{2}$ regions in the middle of the utricle, defined as field 1, field 2, and field 3 (Figure 2(a)). The hair bundle angle was measured based on the position of kinocilium (spectrin-negative on the apical surface), by defining $0^{\circ}$ as the anterior apex and $90^{\circ}$ as the lateral side, and quantified using ImageJ64 (National Institutes of Health, Bethesda, MD, USA). A single kinocilium could be found directly in the electron microscope specimens. Matlab and Screen protractor were used to draw the angle distribution diagram. Statistical analyses were conducted using Prism 8 (GraphPad Software Inc., San Diego, CA, USA). A two-tailed, unpaired Student's $t$-test was used to determine statistical significance. $P<0.05$ was considered significant. Data are shown as mean $\pm \mathrm{SD}$. 


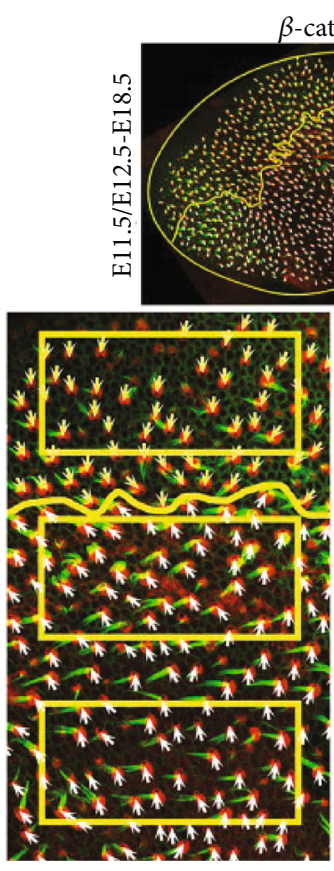

$\beta$-cat $\mathrm{KO}^{\mathrm{fl} / \mathrm{fl}}$
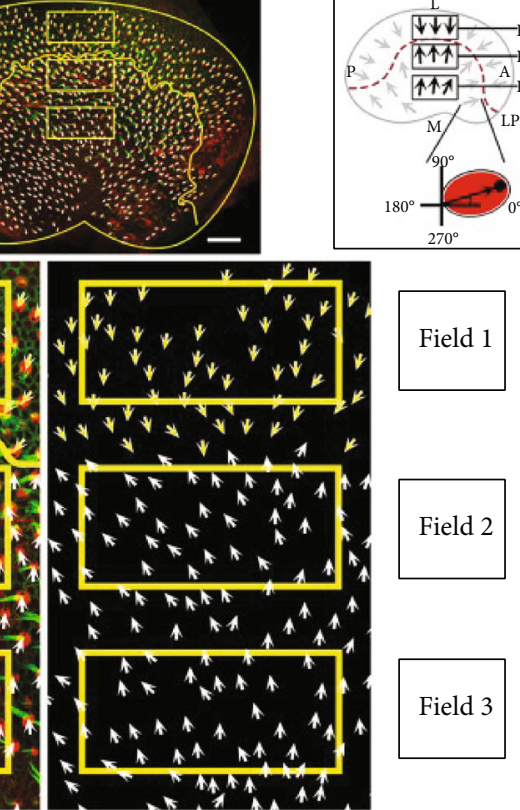

(a)
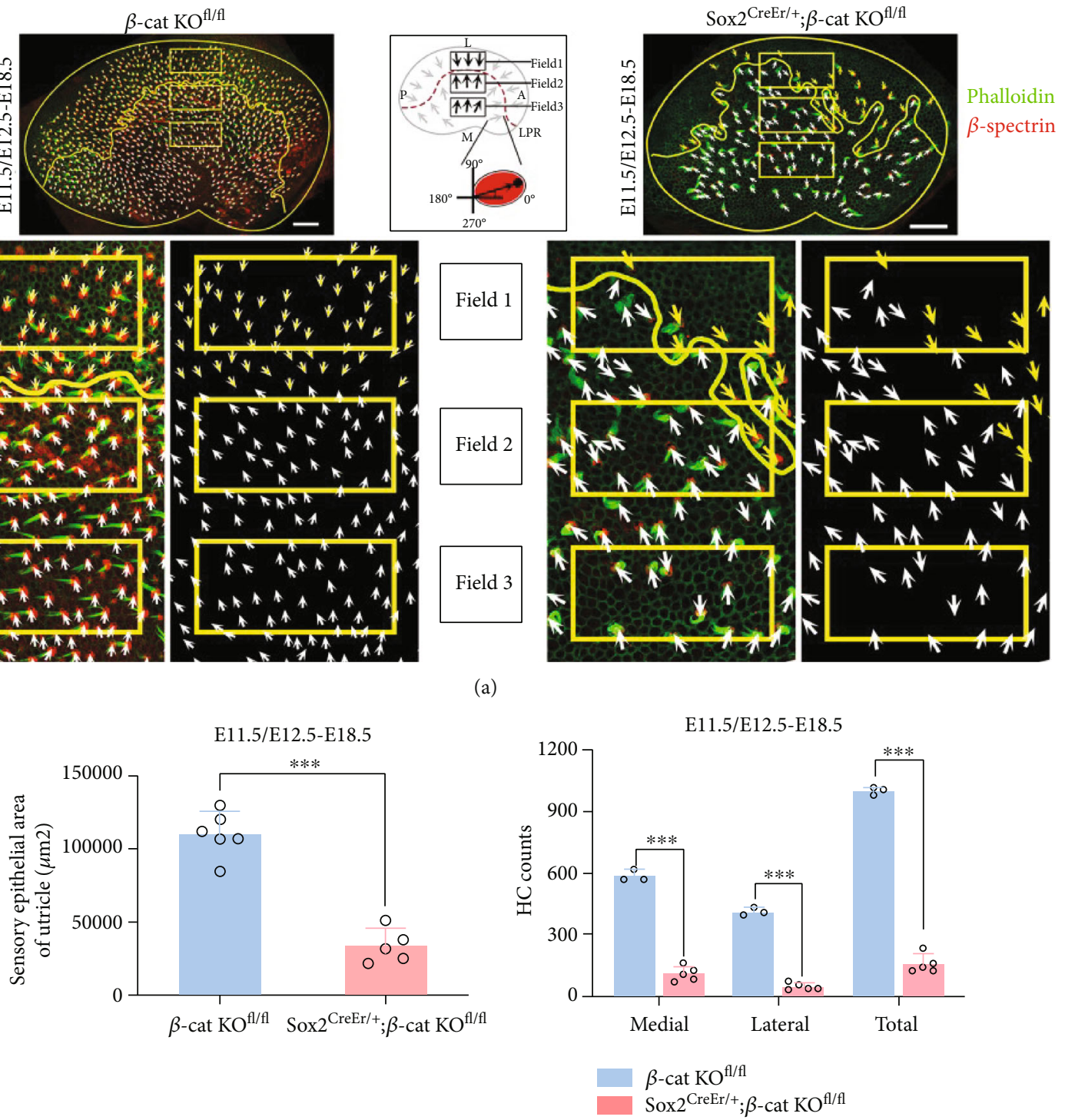

(b)

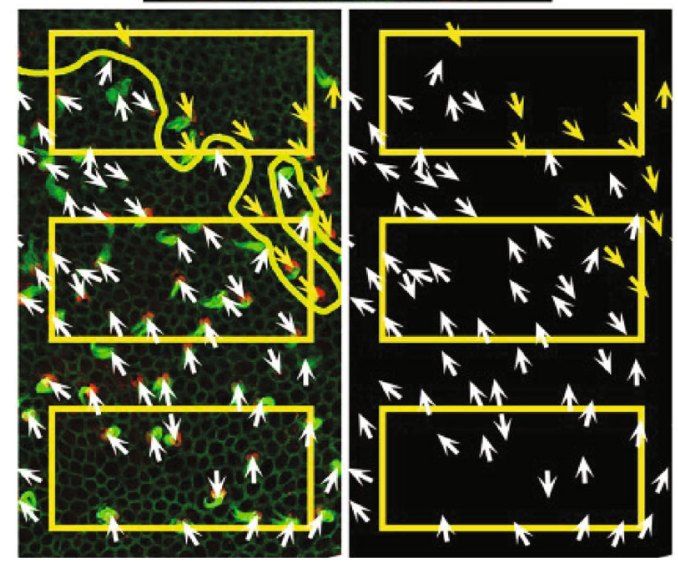

(c)

Figure 2: Continued. 


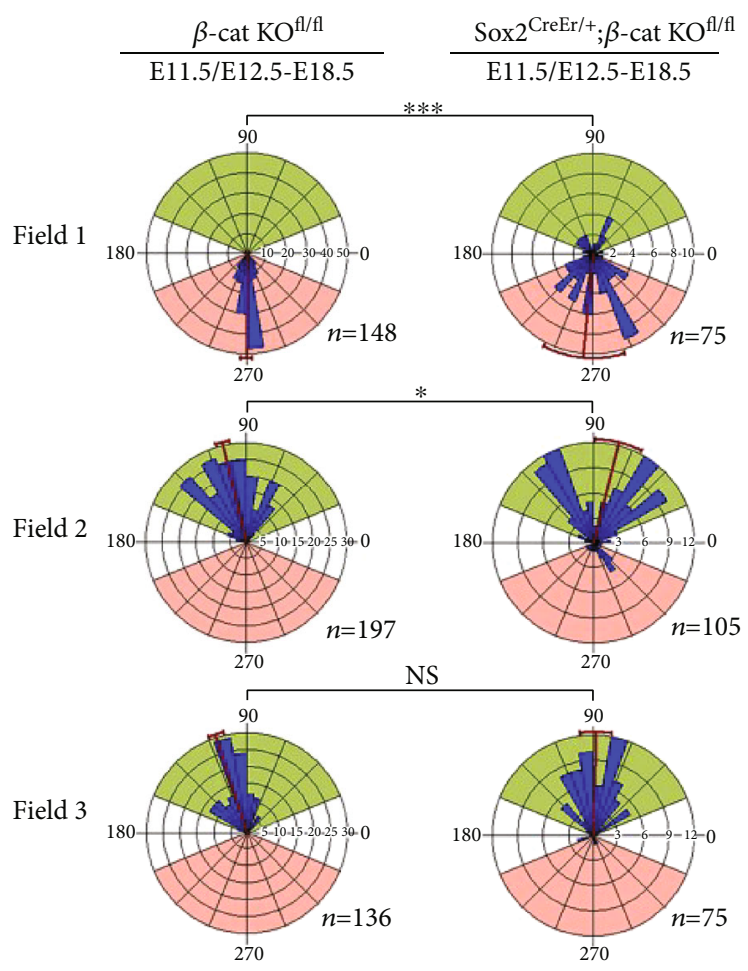

(d)

FIgURE 2: Knockout (KO) $\beta$-catenin in Sox2 positive cells influenced the orientation and number of vestibular hair cells. (a) Compared to the controls (left), Sox $2^{\mathrm{CreER} /+}$ and $\beta$-catenin ${ }^{\mathrm{fl} / \mathrm{fl}}$ (tamoxifen given at E11.5 and E12.5) utricles (right) showed a significant decrease in sensory epithelium area and HC number and a different extent of hair bundle orientation irregularity in fields 1-3. The schematic diagram in the middle black box defining regions of the utricle and illustrating the hair bundle polarity pattern. (b) Quantification of the sensory epithelium area of control and $\beta$-catenin KO utricles (controls, $n=6$; mutants, $n=5$ ). (c) Quantification of HC density on the lateral and medial sides of the LPR and the whole sensory epithelium, from control and $\beta$-catenin KO utricles (controls, $n=3$; mutants, $n=5$ ). (d) Circular histograms of the hair bundle orientation in field 1, field 2, and field 3 for control and $\beta$-catenin KO utricles (controls, $n=3$; mutants, $n=5)$. LPR: line of polarity reversal; L: lateral; M: medial; A: anterior; P: posterior. ${ }^{*} P<0.05,{ }^{* * *} P<0.001$.

\section{Result}

3.1. Wnt Signaling Is Active in the E13.5 Utricle. Our previous studies showed that E13.5 is a critical period for the formation of polarity in the utricle [28]. Two clusters of HCs with opposite cilia began to appear in the utricle, gradually forming LPR. The Wnt signaling pathway is important for embryonic development and plays a variety of roles at different time points during development. Therefore, the primary purpose of our study is to understand the expression of Wnt pathway-related genes in the early utricle.

Wnt5a, Wnt7a, Wnt11, $\beta$-catenin, and Frizzled are important genes in the canonical/noncanonical Wnt signaling pathway. We detected these genes in the utricle by RTPCR. Our research shows that these genes were expressed in the utricle of E13.5 mice (Figure 3(a)).

3.2. Changes of Wnt Signaling in the Early Development of the Utricle. To explore changes in Wnt signaling pathway activity during early development of the utricle, RNA from the utricle of P1 and E13.5 was extracted for RNA-Seq analysis.

Our results showed that Wnt pathway-related genes were differentially expressed. Compared to E13.5,the upregulated genes in P1 were Apc, Axin1, Axin2, Camk2d, Rac1, Fzd1,
Fzd6, Dvl1, Wif1, Smad4, Wnt7a, Nkd1, Sfrp2, Ryk, Siah1a, Camk2g, Ctnnd2, Rspo3, Ppp3cb, Map3k7, Tcf7l1, Prkacb, Porcn, Cxxc4, Prkaca, Vangl1, Ppp3ca, Senp2, Prickle2, Nfatc1, Nfatc3, Nlk, Fzd4, Rock2, Prkcd, Prkce, Prkcz, Ctbp2, Prkci, Bambi, Mapk8, Csnk2a2, Frat1, Prickle1, Ppard, Gsk3a, Tle1, Tle4, Gpc1, Pygo2, Sdc3, and Sdc4. The downregulated genes included Camk2b, Psen1, Ruvbl1, Trp53, Ccnd1, Cacybp, Rhoa, Sfrp5, Csnk1g1, Csnk2b, Sdc1, Bcl9, Gpc2, Gpc3, Lgr5, Ccnd2, Cby1, Dkk2, Rbx1, Myc, Wnt7b, Ppp3r1, Frat2, Serpinf1, and Rhoc (Figure 3(b)). There were some representative genes of canonical Wnt signaling, such as Apc, Axin2, and Wif1. The protein interaction network of Wnt pathway-related genes showed their ranking (Figure 3(c)). Finally, we used RT-qPCR for verification and found that some canonical Wnt pathway-related genes differed in the expression levels of E13.5 and P1 (Figure 3(d)).

3.3. Conditional Knock-out $\beta$-Catenin Affects the Number and Polarity of Vestibular HCs. The areas of utricle maculae in the control and experimental groups were 96,326.63 $\pm 36,029.13 \mu \mathrm{m}^{2} \quad(n=7)$ and $33,824.00 \pm 10,515.93 \mu \mathrm{m}^{2}$ $(n=5)$, respectively, $P<0.05$ (Figure $2(\mathrm{~b})$ ). In the control group $(n=3)$, the total number of HCs was $995.67 \pm$ 14.61, with $585.33 \pm 33.40$ and $410.33 \pm 15.41$ in the 


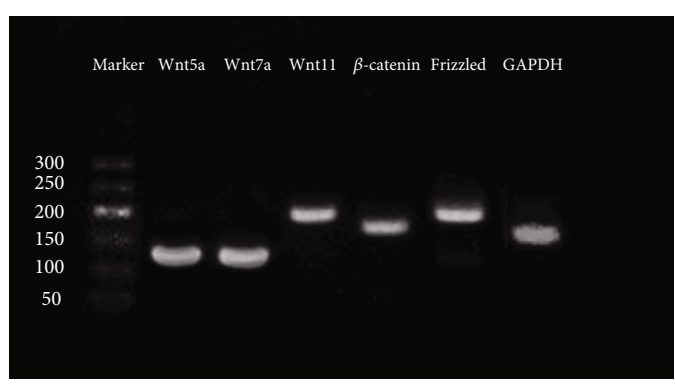

(a)

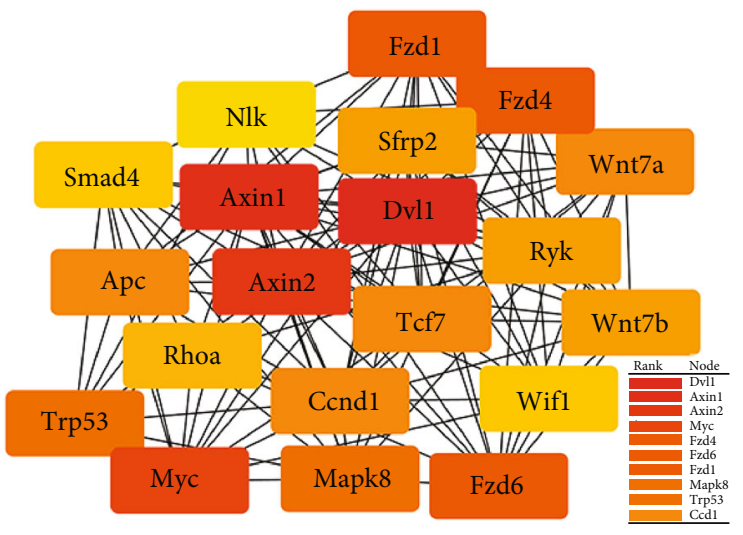

(c)

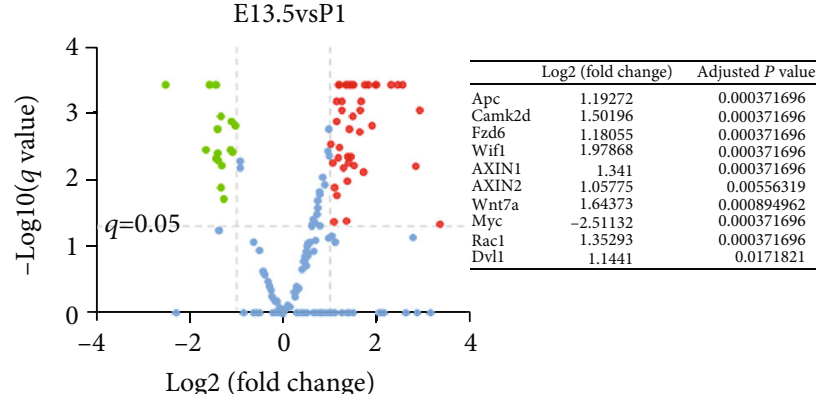

(b)

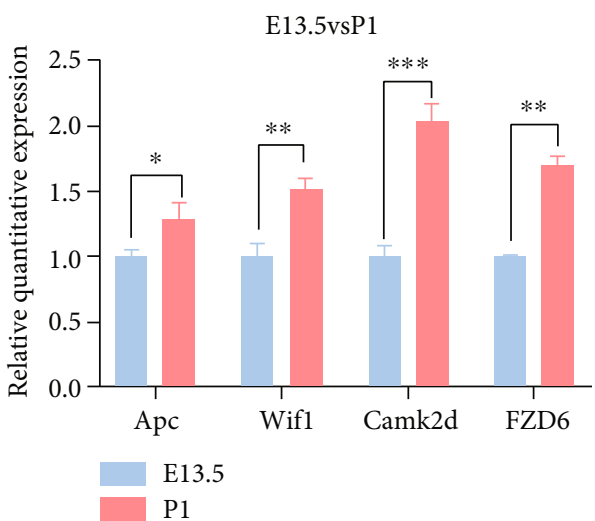

(d)

FIgURE 3: Wnt signaling pathway is active in the E13.5 utricle and changes during utricle development. (a) RT-PCR shows that Wnt5a, Wnt7a, Wnt11, $\beta$-catenin, and Frizzled were expressed in the utricle of E13.5 mice. (b) Differentially expressed Wnt pathway-related genes between the E13.5 and P1 utricle. The red dots represent the upregulated genes based on an adjusted $P$ value $<0.05$ and $\mid \log 2$ (fold change) $\mid>1$; the green dots represent the downregulated genes based on an adjusted $P$ value $<0.05$ and $\mid \log 2$ (fold change) $\mid>1$; the blue spots represent genes with no significant difference in expression. (c) PPI network of E13.5 and P1 utricle. Circles represent genes. Lines represent interactions between gene-encoded proteins, and the top 10 rankings are listed. (d) The relative levels of APC, Wif1, Camk2d, and Fzd6 were analysed by RT-qPCR. The data presented are based on at least three independent experiments. The data are presented as the mean \pm SEM. ${ }^{*} P<0.05,{ }^{* *} P<0.01,{ }^{* * *} P<0.001$.

medial and lateral regions, respectively, whereas in the experimental group $(n=5)$, the total number of HCs was $156.80 \pm 40.09$ with $109.00 \pm 30.25$ and $47.80 \pm 15.46$ in the medial and lateral regions, respectively, $P<0.05$ (Figure 2(c)). As in the control group, $\beta$-catenin knockout mice still showed relatively clear LPRs, with the HCs on both sides having roughly normal directions (Figure 2(a)). However, it is worth noting that the direction of some HCs near LPR, in filed 1 and filed 2 regions, was disordered (Figure 2(d)).

3.4. Regulation of Wnt Signaling In Vitro Leads to Polarity Changes in the Utricle. To determine the role of the Wnt signaling pathway in the polarity of HCs in the utricle, we regulated Wnt signaling pathway activity in vitro using a Wnt/ $\beta$-catenin inhibitor (IWP2) and a Wnt/ $\beta$ catenin activator ( $\mathrm{LiCl}$ ) [29]. According to daily observations of the growth of the utricle, the final culture concentrations were 2.5 and $1.0 \mu \mathrm{M} / \mathrm{mL}$ for $\mathrm{LiCl}$ and IWP2, respectively.

Using a scanning electron microscope, we found that the polarity of the utricle was affected by treatment with the inhibitor or activator. Compared to the control group (Figures 4(a)and 4(b)), the position of the LPR in the acti- vation group was changed (Figure 4, A' and B'), and the polarity of HCs on the lateral and medial side of the LPR was disturbed after adding the activator. In the inhibition group, the polarity of HCs on the lateral and medial sides of the LPR was disturbed (Figure 4, A" and B"). Circular histograms of the hair bundle orientation also showed the difference (Figure 4(c)).

\subsection{Axin2 Is Decreased in Both the Activation and Inhibition} Groups in mRNA. According to RNA-seq results and common Wnt pathway genes, RT-qPCR was performed to detect canonical Wnt signaling pathway-related genes (Apc, Wif1, and Axin2) and PCP core genes (Prickle2 and Vangl2) in cultured utricle in vitro to explore the possible mechanism of polarity change (Figure 5). We found that the expression of Wifl decreased in the inhibitor group and increased in the activator group, while the expression of APC decreased in the activator group. PCP core genes (Prickle2 and Vangl2) did not change significantly in both groups. Interestingly, the expression of Axin2 was decreased in both groups, indicating that Axin2 may be an important role affecting the polarity of the utricle. 

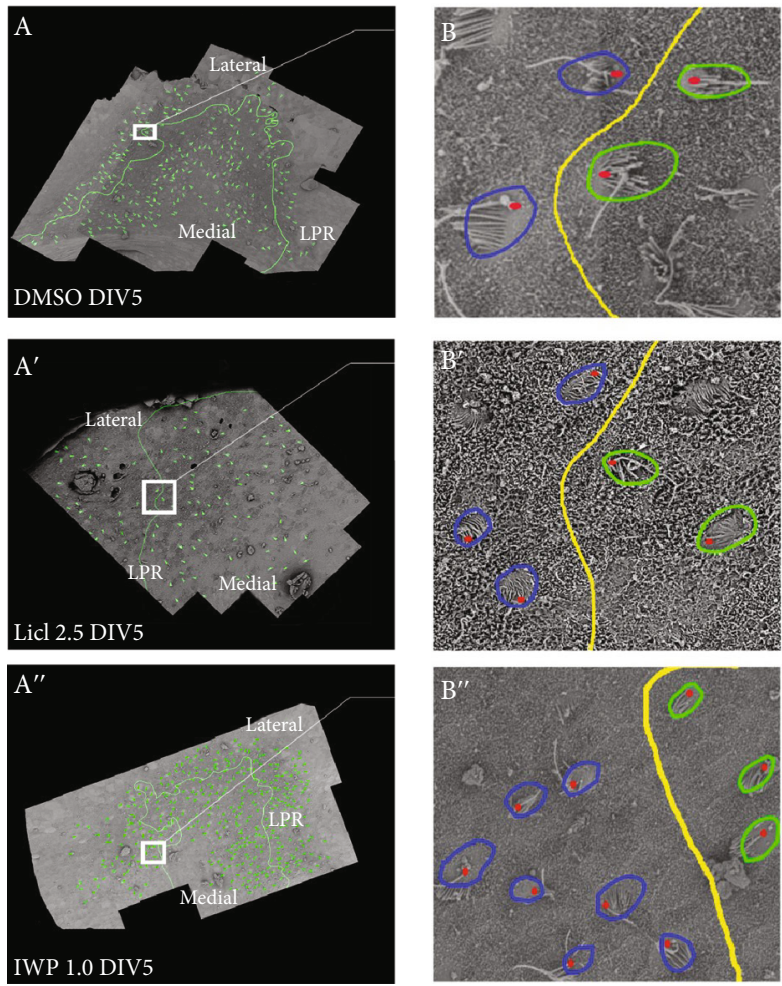

(a)
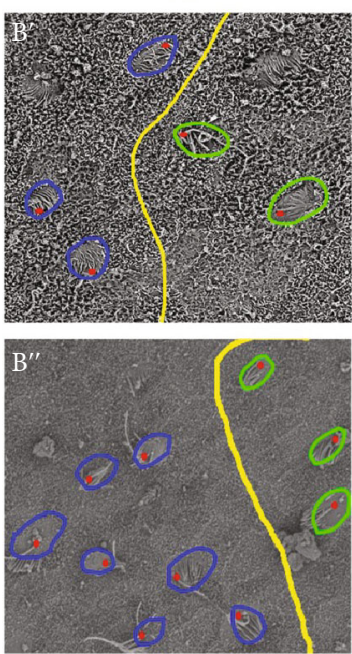

(b)
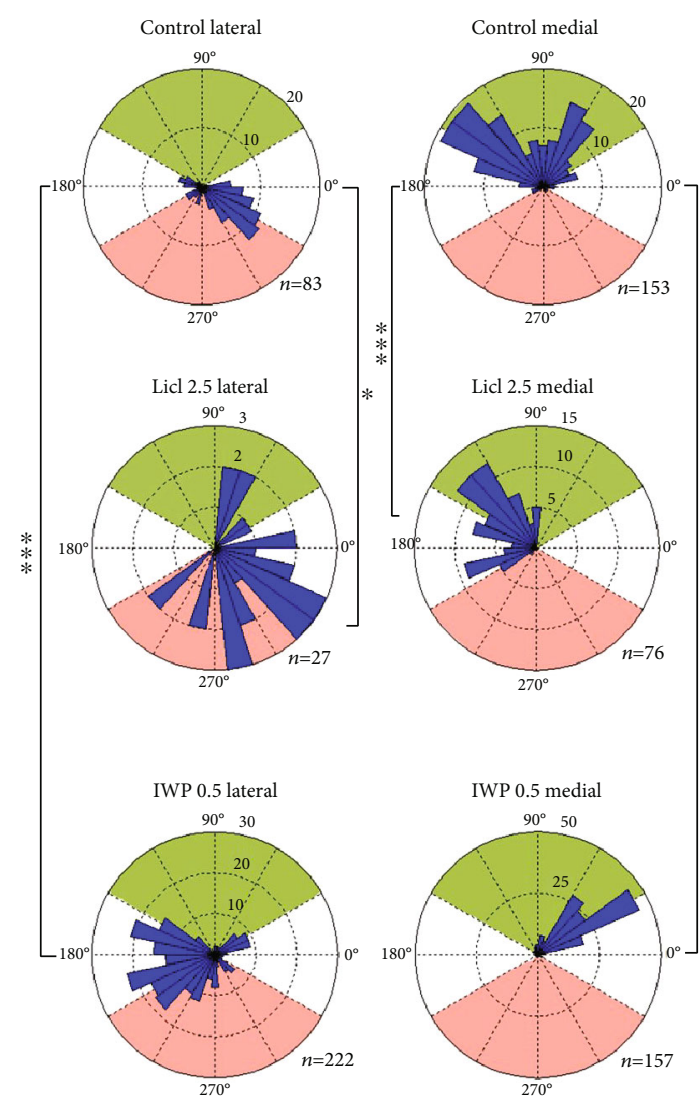

(c)

FIGURE 4: Regulation of Wnt signaling in vitro. (a) A' and A" correspond to the control, activation, and inhibition groups, respectively. DMSO, LiCl, and IWP2 were added into Petri dishes with E13.5 utricles and cultured for 5 days. Scanning electron microscopy showed the polarity and line of polarity reversal (LPR) of the hair cells (HCs) in the control group. The green line represents the LPR, and the green arrow represents the direction of the HC polarity. (b) B' and B" are the white boxes in (a) A' and A," respectively; the green circles represent the HCs on the medial side of the LPR, and the blue circles represent the HCs on the lateral side of the LPR; the red dots represent the position of the kinocilium. Circular histograms of the hair bundle orientation in lateral and medial regions for the control, activator, and inhibitor utricles. ${ }^{*} P<0.05,{ }^{* * *} P<0.001$.

\section{Discussion}

The utricle is the organ that senses balance. Proper utricle function depends on the polarization of hair bundles located on the HC surface. This cellular polarization has also been found in many epithelial cell types, including in the respiratory airway and lateral ventricles of the brain. The planar polarity of the utricle was described at three levels: subcellular, cellular, and tissue-wide [8]. In the inner ear, tissue polarity exists only in the vestibular organ. HCs are oriented such that their stereociliary bundles point toward the LPR in the mammalian utricle. LPR was intact in mouse embryos at E15 [30]. Our previous studies found that the HCs in the lateral extrastriolar region (E13.5) appear later than in the medial extrastriolar regions (E11.5). Thus, E13.5 is a critical period for the formation of polarity [28]. In this study, we treated utricles at or before E13.5, either by adding inhibitor or activator in vitro or by knocking out $\beta$-catenin in vivo. The results showed that the canonical Wnt signaling pathway played a role in polarity development of the utricle.

Wnt signaling is a marker of embryonic development and has multiple functions at a number of developmental time points. The Wnt signaling pathway includes both canonical and noncanonical forms. The Wnt signaling pathway has been studied in the early development of the cochlea. The Wnt signaling receptors (Fzd 1, 2, 3, 4, 6, Ryk, Ror2, and Lgr5) can be detected in the cochlea of mice at E14.5 [3136]. The expression of Wnt7a, Wnt5a, Wnt2, Wnt10b, Wnt4, Wnt7b, Wnt8, and Wnt11 could be detected in the cochlea at E15 and E17 [37], and studies suggested a potential alternation between canonical and noncanonical signaling pathways [38, 39]. Our study also revealed both canonical and noncanonical Wnt pathway-related genes in the utricle during early development, and most Wnt pathway genes were also different between the two time periods of E13.5 and $\mathrm{P} 1$. These greatly altered genes may contribute to valid proliferation, differentiation, or innervation. When $\mathrm{LiCl}$ was added to activate the Wnt pathway in vitro, the proliferation domain of Sox2-positive cells in the cochlea (E12.5) was significantly expanded, indicating that the canonical Wnt pathway regulates the proliferation and differentiation of HCs during early development of the cochlea [14]. Furthermore, overexpression of $\beta$-catenin initiated proliferation of sensory precursors cells within the cochlear sensory 


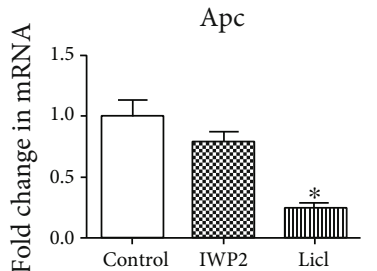

(a)

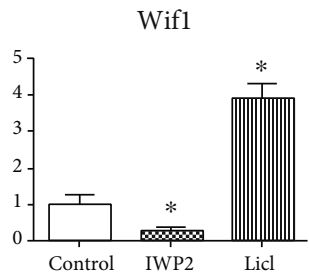

(c)

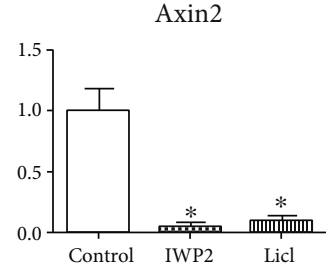

(b)

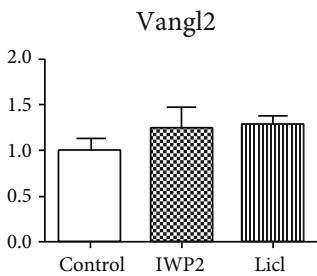

(d)

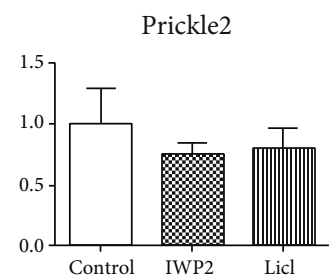

(e)

FIgURE 5: Analysis of Wnt pathway-related genes in vitro treatment at RNA level. (a) Compared with the control group, the expression of Apc was decreased with Licl treatment, and there was no statistical difference with IWP2 treatment. (b) The expression of Axin2 was decreased with IWP2 treatment or Licl treatment. (c) The expression of Wif1 was decreased with IWP2 treatment and was increased with Licl treatment. (d, e) Vangl2 and Prickle2 were no statistical difference with IWP2 treatment or Licl treatment, compared with the control group. ${ }^{*} P<0.05$.

epithelium [40]. Therefore, we hypothesized that the Wnt pathway might play the same role in the early development of the utricle. This was confirmed in vivo when we conditionally knocked out $\beta$-catenin that the number of utricle HCs was affected in the mic. In addition, we found changes in the polarity of hair cells.

Indeed, the Wnt pathway also plays an important role in inner ear polarity regulation. The polarity of $\mathrm{HCs}$ in the cochlea is mainly reflected in their convergence and extension, an identical ciliary direction among all $\mathrm{HCs}$, and a Vshaped stereociliary bundle with a uniform direction [41]. In vestibular organs, the stereocilia arrangement and kinocilium displacement are in accordance with a subcellular polarity. HCs are surrounded by supporting cells, and all HCs showed directional coordination in this study. Regarding tissue polarity, cilia bundles of HCs are present on both sides of the LPR face, or move away from the LPR. The role of the Wnt/PCP pathway, a noncanonical Wnt pathway, in inner ear polarity has been studied extensively [34, 42-44]. The noncanonical Wnt/PCP signaling pathway mainly involves Wnt proteins, such as Wnt4, Wnt5A, and Wnt11 [45]. These proteins activate the DEP domain of the Dsh protein, to in turn activate downstream homologous paleogenesis by PCP core proteins, independent of the accumulation of $\beta$-catenin. Dislocation of the hair bundle is a common phenotype of Wnt/PCP pathway mutants in the inner ear. The cochlea of the Wnt5a mutant is wider and shorter, with stereociliary bundle misorientation in accordance with a subcellular polarity [35]. After knocking out Vangl or Frizzled, which are the core PCP genes, we observed a disturbance in the polarity of neighbouring cells that appeared relatively consistent, while its subcellular polarity remained intact $[34,46]$. In addition, Testin, Celsr1, and Pk2 affect the PCP protein distribution in the vestibule according to the cellular polarity $[44,47,48]$. It is generally believed that the PCP axis is established by noncanonical Wnt signals for tissue polarity. Therefore, it is worth investigating whether canonical Wnt signals play a role in the polarity of the utricle.

Our results showed that the Wnt genes expressed on different days in the utricle were closely related to canonical Wnt/ $\beta$-catenin pathway, such as Axin2. Thus, we suspect that the canonical Wnt pathway also plays an important role in regulating polarity. The canonical Wnt pathway has been implicated in the regulation of polarity in other organs and animals. Local activation of the canonical Wnt pathway regulates neuronal polarity and axonal outgrowth [49]. In addition, our previous study found that Rack 1 is involved in cell membrane localization of the polarity core protein Vangl2, which is associated with zebrafish planar cell polarity formation, primarily by antagonizing the $\mathrm{Wnt} / \beta$-catenin signaling pathway [50]. $\beta$-catenin is the core protein in the canonical Wnt pathway that controls the expression of downstream target genes. A mouse with conditional knockout of $\beta$ catenin in the dorsal neural folds will exhibit spina bifida 
aperta, caudal axis bending, and tail truncation [51]. Similar polarity changes occurred in mouse kidneys with conditional knockout of $\beta$-catenin [52]. However, there are few studies on the canonical Wnt pathway in the context of polarity development in the inner ear, especially in the vestibule. Ankrd6 is asymmetrically distributed in the utricle and regulates polarity by inhibiting the canonical $\mathrm{Wnt} / \beta$-catenin signaling pathway [53]. Some researchers suggested that the $\mathrm{Wnt} / \beta$-catenin pathway is important in the ground hypothesis, which was suggested to explain the formation of LPR in the vestibule [54]; however, this has not been verified in mammals. Sox 2 is expressed throughout the developing nervous system and plays a role in hair cell type formation [55]. We knocked out $\beta$-catenin in Sox2-positive cells and found that the utricle polarity was subsequently affected: the directions of some HCs were disordered near the LPR in the lateral and striolar regions. The changed polarity was also found after activating and inhibiting canonical Wnt pathways in vitro. The changed polarity was also found after activating and inhibiting canonical Wnt pathways in vitro, and PCP core genes (Vangl2 and Prickle2) were no statistical difference with IWP2 treatment or Licl treatment at the RNA level, compared with the control group. However, Axin2 unexpectedly decreased in the two treatment groups. Axin2, a scaffolding protein of glycogen synthase kinase 3 , is a negative regulator of Wnt signaling. In addition, Axin2 feedback loop is important with many studies in Wnt pathway, which could help to limit Wnt-initiated signal [56]. Axin2 is involved in the development of the permanent teeth, hair, and eye brows and regulates calvarial suture closure in skull development $[57,58]$. In the study of hair cells, a subset of hair cells is derived from Axin2-expressing tympanic border cells, and Axin 2 cells were able to differentiate into hair celllike cells [59]. These findings may suggest that Axin2 has the value in the polarity of utricle, which needs further study to elucidate.

\section{Conclusion}

In this study, we investigated expression changes of Wnt pathway-related genes during early development of the mouse utricle. In conditional knockout of $\beta$-catenin in vivo, our data showed the main effect is on hair cell number and may reveal an impact on stereociliary bundle orientation. Combined with the in vitro results, we believe that the canonical Wnt pathway is important for controlling HC polarity during mammalian utricle development. Our findings may stimulate future studies on vestibule polarity development.

\section{Data Availability}

All data used to support the findings of this study are available from the corresponding author upon request.

\section{Conflicts of Interest}

The authors have declared no conflict of interest.

\section{Authors' Contributions}

Di Deng, Xiaoqing Qian, and Binjun Chen have contributed equally to this work and share first authorship.

\section{Acknowledgments}

This study was supported by the National Natural Science Foundation of China (NSFC) (Grant nos. 81771017, 81970880, and 81970889).

\section{References}

[1] Y. Liu, J. Qi, X. Chen et al., "Critical role of spectrin in hearing development and deafness," Science Advances, vol. 5, no. 4, p. eaav7803, 2019.

[2] Y. Wang, J. Li, X. Yao et al., "Loss of CIB2 causes profound hearing loss and abolishes mechanoelectrical transduction in mice," Frontiers in Molecular Neuroscience, vol. 10, p. 401, 2017.

[3] C. Zhu, C. Cheng, Y. Wang et al., "Loss of ARHGEF6 causes hair cell stereocilia deficits and hearing loss in mice," Frontiers in Molecular Neuroscience, vol. 11, p. 362, 2018.

[4] H. Zhou, X. Qian, N. Xu et al., "Disruption of Atg7-dependent autophagy causes electromotility disturbances, outer hair cell loss, and deafness in mice," Cell Death \& Disease, vol. 11, no. 10, p. 913, 2020.

[5] P. G. Gillespie and U. Müller, "Mechanotransduction by hair cells: models, molecules, and mechanisms," Cell, vol. 139, no. 1, pp. 33-44, 2009.

[6] J. Qi, Y. Liu, C. Chu et al., "A cytoskeleton structure revealed by super-resolution fluorescence imaging in inner ear hair cells," Cell Discovery, vol. 5, no. 1, p. 12, 2019.

[7] J. Qi, L. Zhang, F. Tan et al., "Espin distribution as revealed by super-resolution microscopy of stereocilia," American Journal of Translational Research, vol. 12, no. 1, pp. 130-141, 2020.

[8] M. R. Deans, "A balance of form and function: planar polarity and development of the vestibular maculae," Seminars in Cell \& Developmental Biology, vol. 24, no. 5, pp. 490-498, 2013.

[9] S. Zhang, D. Liu, Y. Dong et al., "Frizzled-9+ supporting cells are progenitors for the generation of hair cells in the postnatal mouse cochlea," Frontiers in Molecular Neuroscience, vol. 12, p. 184, 2019.

[10] Y. Chen, X. Lu, L. Guo et al., "Hedgehog signaling promotes the proliferation and subsequent hair cell formation of progenitor cells in the neonatal mouse cochlea," Frontiers in Molecular Neuroscience, vol. 10, p. 426, 2017.

[11] X. Lu, S. Sun, J. Qi et al., "Bmil regulates the proliferation of cochlear supporting cells via the canonical Wnt signaling pathway," Molecular Neurobiology, vol. 54, no. 2, pp. 1326-1339, 2017.

[12] J. Wu, W. Li, C. Lin et al., "Co-regulation of the Notch and Wnt signaling pathways promotes supporting cell proliferation and hair cell regeneration in mouse utricles," Scientific Reports, vol. 6, no. 1, p. 29418, 2016.

[13] T. Wang, R. Chai, G. S. Kim et al., "Lgr5+ cells regenerate hair cells via proliferation and direct transdifferentiation in damaged neonatal mouse utricle," Nature Communications, vol. 6, no. 1, p. 6613, 2015.

[14] S. Zhang, Y. Zhang, Y. Dong et al., "Knockdown of Foxg1 in supporting cells increases the trans-differentiation of 
supporting cells into hair cells in the neonatal mouse cochlea," Cellular and Molecular Life Sciences, vol. 77, no. 7, pp. 14011419, 2020.

[15] S. Zhang, R. Qiang, Y. Dong et al., "Hair cell regeneration from inner ear progenitors in the mammalian cochlea," American Journal of Stem Cells, vol. 9, no. 3, pp. 25-35, 2020.

[16] B. C. Cox, R. Chai, A. Lenoir et al., "Spontaneous hair cell regeneration in the neonatal mouse cochlea in vivo," Development, vol. 141, no. 4, pp. 816-829, 2014.

[17] Y. Zhang, S. Zhang, Z. Zhang et al., "Knockdown of Foxg1 in Sox9+ supporting cells increases the trans-differentiation of supporting cells into hair cells in the neonatal mouse utricle," Aging (Albany NY), vol. 12, no. 20, pp. 19834-19851, 2020.

[18] W. Li, D. You, Y. Chen, R. Chai, and H. Li, "Regeneration of hair cells in the mammalian vestibular system," Frontiers in Medicine, vol. 10, no. 2, pp. 143-151, 2016.

[19] R. van Amerongen and R. Nusse, "Towards an integrated view of Wnt signaling in development," Development, vol. 136, no. 19, pp. 3205-3214, 2009.

[20] L. Liu, Y. Chen, J. Qi et al., "Wnt activation protects against neomycin-induced hair cell damage in the mouse cochlea," Cell Death \& Disease, vol. 7, no. 3, pp. e2136-e2136, 2016.

[21] Z. H. He, S. Y. Zou, M. Li et al., "The nuclear transcription factor FoxG1 affects the sensitivity of mimetic aging hair cells to inflammation by regulating autophagy pathways," Redox Biology, vol. 28, p. 101364, 2020.

[22] Y. Ding, W. Meng, W. Kong, Z. He, and R. Chai, "The role of FoxG1 in the inner ear," Frontiers in Cell and Development Biology, vol. 8, p. 614954, 2020.

[23] Z. He, Q. Fang, H. Li et al., "The role of FOXG1 in the postnatal development and survival of mouse cochlear hair cells," Neuropharmacology, vol. 144, pp. 43-57, 2019.

[24] S. Gao, C. Cheng, M. Wang et al., "Blebbistatin Inhibits Neomycin-Induced Apoptosis in Hair Cell-Like HEI-OC-1 Cells and in Cochlear Hair Cells," Frontiers in Cellular Neuroscience, vol. 13, p. 590, 2019.

[25] W. Liu, X. Xu, Z. Fan et al., "Wnt signaling activates TP53induced glycolysis and apoptosis regulator and protects against cisplatin-induced spiral ganglion neuron damage in the mouse cochlea," Antioxidants \& Redox Signaling, vol. 30, no. 11, pp. 1389-1410, 2019.

[26] C. Cheng, Y. Wang, L. Guo et al., "Age-related transcriptome changes in Sox2+ supporting cells in the mouse cochlea," Stem Cell Research \& Therapy, vol. 10, no. 1, p. 365, 2019.

[27] T. Ohyama, O. A. Mohamed, M. M. Taketo, D. Dufort, and A. K. Groves, "Wnt signals mediate a fate decision between otic placode and epidermis," Development, vol. 133, no. 5, pp. 865-875, 2006.

[28] X. Yang, X. Qian, R. Ma et al., "Establishment of planar cell polarity is coupled to regional cell cycle exit and cell differentiation in the mouse utricle," Scientific Reports, vol. 7, no. 1, p. 43021, 2017.

[29] Z. Li, S. Chen, S. Chen, D. Huang, K. Ma, and Z. Shao, "Moderate activation of $\mathrm{Wnt} / \beta$-catenin signaling promotes the survival of rat nucleus pulposus cells via regulating apoptosis, autophagy, and senescence," Journal of Cellular Biochemistry, vol. 120, no. 8, pp. 12519-12533, 2019.

[30] K. Denman-Johnson and A. Forge, "Establishment of hair bundle polarity and orientation in the developing vestibular system of the mouse," Journal of Neurocytology, vol. 28, no. 10/11, pp. 821-835, 1999.
[31] R. Chai, A. Xia, T. Wang et al., "Dynamic expression of Lgr5, a Wnt target gene, in the developing and mature mouse cochlea," Journal of the Association for Research in Otolaryngology, vol. 12, no. 4, pp. 455-469, 2011.

[32] M. L. Macheda, W. W. Sun, K. Kugathasan et al., "The Wnt receptor Ryk plays a role in mammalian planar cell polarity signaling," The Journal of Biological Chemistry, vol. 287, no. 35, pp. 29312-29323, 2012.

[33] H. Yu, P. M. Smallwood, Y. Wang, R. Vidaltamayo, R. Reed, and J. Nathans, "Frizzled 1 and frizzled 2 genes function in palate, ventricular septum and neural tube closure: general implications for tissue fusion processes," Development, vol. 137, no. 21, pp. 3707-3717, 2010.

[34] Y. Wang, N. Guo, and J. Nathans, "The role of Frizzled3 and Frizzled6 in neural tube closure and in the planar polarity of inner-ear sensory hair cells," The Journal of Neuroscience, vol. 26, no. 8, pp. 2147-2156, 2006.

[35] D. Qian, C. Jones, A. Rzadzinska et al., "Wnt5a functions in planar cell polarity regulation in mice," Developmental Biology, vol. 306, no. 1, pp. 121-133, 2007.

[36] Y. Wang, D. Huso, H. Cahill, D. Ryugo, and J. Nathans, "Progressive cerebellar, auditory, and esophageal dysfunction caused by targeted disruption of the frizzled-4 gene," The Journal of Neuroscience, vol. 21, no. 13, pp. 4761-4771, 2001.

[37] A. Dabdoub, M. J. Donohue, A. Brennan et al., "Wnt signaling mediates reorientation of outer hair cell stereociliary bundles in the mammalian cochlea," Development, vol. 130, no. 11, pp. 2375-2384, 2003.

[38] A. J. Mikels and R. Nusse, "Purified Wnt5a protein activates or inhibits beta-catenin-TCF signaling depending on receptor context," PLoS Biology, vol. 4, no. 4, article e115, 2006.

[39] J. D. Berndt, A. Aoyagi, P. Yang, J. N. Anastas, L. Tang, and R. T. Moon, "Mindbomb 1, an E3 ubiquitin ligase, forms a complex with RYK to activate Wnt/ $\beta$-catenin signaling," The Journal of Cell Biology, vol. 194, no. 5, pp. 737-750, 2011.

[40] R. Chai, B. Kuo, T. Wang et al., "Wnt signaling induces proliferation of sensory precursors in the postnatal mouse cochlea," Proceedings of the National Academy of Sciences of the United States of America, vol. 109, no. 21, pp. 8167-8172, 2012.

[41] A. Li, J. Xue, and E. H. Peterson, "Architecture of the mouse utricle: macular organization and hair bundle heights," Journal of Neurophysiology, vol. 99, no. 2, pp. 718-733, 2008.

[42] X. Lu, A. G. Borchers, C. Jolicoeur, H. Rayburn, J. C. Baker, and M. Tessier-Lavigne, "PTK7/CCK-4 is a novel regulator of planar cell polarity in vertebrates," Nature, vol. 430, no. 6995, pp. 93-98, 2004.

[43] H. Yin, C. O. Copley, L. V. Goodrich, and M. R. Deans, “Comparison of phenotypes between different vangl2 mutants demonstrates dominant effects of the Looptail mutation during hair cell development," PLoS One, vol. 7, no. 2, article e31988, 2012.

[44] J. S. Duncan, M. L. Stoller, A. F. Francl, F. Tissir, D. Devenport, and M. R. Deans, "Celsr1 coordinates the planar polarity of vestibular hair cells during inner ear development," Developmental Biology, vol. 423, no. 2, pp. 126-137, 2017.

[45] H. Peradziryi, N. S. Tolwinski, and A. Borchers, "The many roles of PTK7: a versatile regulator of cell-cell communication," Archives of Biochemistry and Biophysics, vol. 524, no. 1, pp. 71-76, 2012.

[46] M. L. Stoller, O. Roman Jr., and M. R. Deans, "Domineering non-autonomy in Vangl1;Vangl2 double mutants 
demonstrates intercellular PCP signaling in the vertebrate inner ear," Developmental Biology, vol. 437, no. 1, pp. 17-26, 2018.

[47] D. D. Ren, M. Kelly, S. M. Kim, C. M. Grimsley-Myers, F. L. Chi, and P. Chen, "Testin interacts with vangl2 genetically to regulate inner ear sensory cell orientation and the normal development of the female reproductive tract in mice," Developmental Dynamics, vol. 242, no. 12, pp. 1454-1465, 2013.

[48] M. R. Deans, D. Antic, K. Suyama, M. P. Scott, J. D. Axelrod, and L. V. Goodrich, "Asymmetric distribution of prickle-like 2 reveals an early underlying polarization of vestibular sensory epithelia in the inner ear," The Journal of Neuroscience, vol. 27, no. 12, pp. 3139-3147, 2007.

[49] E. Stanganello, E. E. Zahavi, M. Burute et al., "Wnt Signaling Directs Neuronal Polarity and Axonal Growth," iScience, vol. 13, pp. 318-327, 2019.

[50] S. Li, R. Esterberg, V. Lachance et al., "Rack1 is required for Vangl2 membrane localization and planar cell polarity signaling while attenuating canonical Wnt activity," Proceedings of the National Academy of Sciences of the United States of America, vol. 108, no. 6, pp. 2264-2269, 2011.

[51] T. Zhao, Q. Gan, A. Stokes et al., " $\beta$-catenin regulates Pax3 and Cdx2 for caudal neural tube closure and elongation," Development, vol. 141, no. 1, pp. 148-157, 2014.

[52] T. D. Marose, C. E. Merkel, A. P. McMahon, and T. J. Carroll, " $\beta$-Catenin is necessary to keep cells of ureteric bud/Wolffian duct epithelium in a precursor state," Developmental Biology, vol. 314, no. 1, pp. 112-126, 2008.

[53] C. Jones, D. Qian, S. M. Kim et al., “_Ankrd6_is a mammalian functional homolog of _Drosophila_planar cell polarity gene _diego_ and regulates coordinated cellular orientation in the mouse inner ear," Developmental Biology, vol. 395, no. 1, pp. 62-72, 2014.

[54] J. L. Green, T. Inoue, and P. W. Sternberg, "Opposing Wnt pathways orient cell polarity during organogenesis," Cell, vol. 134, no. 4, pp. 646-656, 2008.

[55] J. Lu, L. Hu, B. Ye et al., "Increased type I and decreased type II hair cells after deletion of _Sox2_ in the developing mouse utricle," Neuroscience, vol. 422, pp. 146-160, 2019.

[56] E. H. Jho, T. Zhang, C. Domon, C. K. Joo, J. N. Freund, and F. Costantini, "Wnt/beta-catenin/Tcf signaling induces the transcription of Axin2, a negative regulator of the signaling pathway," Molecular and Cellular Biology, vol. 22, no. 4, pp. 1172-1183, 2002.

[57] H.-M. I. Yu, B. Jerchow, T.-J. Sheu et al., “The role of Axin2 in calvarial morphogenesis and craniosynostosis," Development, vol. 132, no. 8, pp. 1995-2005, 2005.

[58] S. K. Macklin Mantia, S. L. Hines, K. L. Chaichana et al., "Case report expanding the germline AXIN2- related phenotype to include olfactory neuroblastoma and gastric adenoma," $B M C$ Medical Genetics, vol. 21, no. 1, p. 161, 2020.

[59] T. A. Jan, R. Chai, Z. N. Sayyid et al., "Tympanic border cells are Wnt-responsive and can act as progenitors for postnatal mouse cochlear cells," Development, vol. 140, no. 6, pp. 1196-1206, 2013. 